\title{
Core-Collapse Supernova Simulations including Neutrino Interactions from the Virial EOS
}

\author{
Evan O'Connor ${ }^{1,2}$, C. J. Horowitz ${ }^{3}$, Zidu Lin $^{3}$ and Sean Couch ${ }^{4,5,6,7}$ \\ ${ }^{1}$ Department of Astronomy and Oskar Klein Centre, Stockholm University, AlbaNova, SE-106 \\ 91 Stockholm, Sweden email: evan.oconnor@astro.su.se \\ ${ }^{2}$ NCSU, Department of Physics, Campus Code 8202, Raleigh, NC, 27695, USA \\ ${ }^{3}$ Center for Exploration of Energy and Matter and Department of Physics, Indiana University, \\ Bloomington, IN 47408, USA \\ ${ }^{4}$ Department of Physics and Astronomy, MSU, East Lansing, MI 48824, USA \\ ${ }^{5}$ Department of Computational Mathematics, Science, and Engineering, Michigan State \\ University, East Lansing, MI 48824, USA \\ ${ }^{6}$ National Superconducting Cyclotron Laboratory, MSU, East Lansing, MI 48824, USA \\ ${ }^{7}$ Joint Institute for Nuclear Astrophysics, MSU, East Lansing, MI 48824, USA
}

\begin{abstract}
Core-collapse supernova explosions are driven by a central engine that converts a small fraction of the gravitational binding energy released during core collapse to outgoing kinetic energy. The suspected mode for this energy conversion is the neutrino mechanism, where a fraction of the neutrinos emitted from the newly formed protoneutron star are absorbed by and heat the matter behind the supernova shock. Accurate neutrino-matter interaction terms are crucial for simulating these explosions. In this proceedings for IAUS 331, SN 1987A, 30 years later, we explore several corrections to the neutrino-nucleon scattering opacity and demonstrate the effect on the dynamics of the core-collapse supernova central engine via two dimensional neutrino-radiation-hydrodynamics simulations. Our results reveal that the explosion properties are sensitive to corrections to the neutral-current scattering cross section at the $10-20 \%$ level, but only for densities at or above $\sim 10^{12} \mathrm{~g} \mathrm{~cm}^{-3}$.
\end{abstract}

Keywords. (stars:) supernovae: general, stars: neutron, methods: numerical, radiative transfer, neutrinos, hydrodynamics, scattering

\section{Introduction}

The observation of neutrinos from SN 1987A confirmed the basic nature of core collapse and highlighted the important role neutrinos play. Since then, 30 years ago, the community has made tremendous progress in elucidating this process. While neutrinos are an extreme sink of energy, and part of the reason that the supernova shock initially stalls, they are also thought to be crucial in reviving the supernova shock via the neutrino mechanism. The neutrino mechanism relies primarily on charged-current heating by electron neutrinos and antineutrinos on the neutrons and protons located behind the shock. This heating gives rise to the so-called gain region, where the heating by the background neutrino field being emitted from deeper regions is larger than the neutrino cooling from the matter. The net result is an increasing internal energy and, if sufficient, the development of an explosion. The amount of neutrino heating depends sensitively on the spectra of the background neutrino radiation fields, both the overall normalization (the luminosity) and its shape (mean energy, root mean squared energy, etc.). 
One of the major difficulties in modeling core-collapse supernovae is the treatment of the neutrino radiation fields. Soon after core bounce, in the core of the protoneutron star, the neutrinos are completely trapped. The mean free path is much shorter than the typical length scales and these neutrinos are in equilibrium with the surrounding matter. However, as one moves away from the core, the densities drop rapidly, the mean free path increases, and the neutrinos decouple from the matter. By $\sim 100 \mathrm{~km}$, most neutrinos are essentially free streaming and only a small percentage $(\sim 5-10 \%)$ will have interactions before exiting the star. It is critical to model the transition away from equilibrium since it is this transition which sets the spectrum of the radiation field responsible for the neutrino heating further out. To model this transition precisely one needs spectral neutrino transport and accurate neutrino opacities.

In this proceedings for IAU Symposium 331, "SN 1987A, 30 years later", we present a series of $2 \mathrm{D}$ core-collapse supernova simulations with spectral neutrino transport where we investigate in detail the effect of varying the neutrino opacities on the core-collapse supernova explosion mechanism. In particular, we use FLASH (Fryxell et al. 2000; Couch and O'Connor 2014; O'Connor and Couch 2015) which is outfitted with an energy-dependent M1 neutrino transport scheme (see below) and an effective general relativistic treatment of gravity. We explore recent corrections to the neutral-current scattering cross sections proposed by Horowitz et al. (2017). We do this generally for four progenitor models, and in detail for one model in particular. Our main conclusion is that the core-collapse supernova explosion mechanism is sensitive to the details of the neutral current cross section at densities of $\sim 10^{12}-10^{13} \mathrm{~g} \mathrm{~cm}^{-3}$. This is currently the limit of model-independent calculations of the neutrino interactions, therefore care and caution must be used when developing models for higher densities.

\section{Methods}

Our FLASH simulations follow the methods of O'Connor \& Couch $(2017$; in prep), a significantly updated version of O'Connor and Couch (2015). Our velocity-dependent neutrino transport scheme is based on the M1 formalism, where we evolve the first two angular moments of the neutrino distribution function, the energy density and the momentum density. We evolve these moments as a function of neutrino species (3 species in total, $\nu_{e}, \bar{\nu}_{e}$, and $\nu_{x}=\left\{\nu_{\mu}, \bar{\nu}_{\mu}, \nu_{\tau}, \bar{\nu}_{\tau}\right\}$ ) and neutrino energy (our simulations use 12 energy groups for each species). We fully take into account gravitational red shifting. We use the LS220 EOS (Lattimer and Swesty 1991). Our neutrino opacities come from NuLib, an open-source neutrino interaction library (O'Connor 2015). The base rates are based on Bruenn (1985) and include corrections for weak magnetism and nuclear recoil (Horowitz 2002). The specific corrections we explore here are based on Horowitz et al. (2017). In Horowitz et al. (2017), we use the Virial equation of state (EOS) to derive model-independent expressions for the axial response function $\left(S_{A}\right)$ of the neutralcurrent, neutrino-nucleon scattering cross section, which is given as

$$
\frac{1}{V} \frac{d \sigma}{d \Omega}=\frac{G_{F}^{2} E_{\nu}^{2}}{16 \pi^{2}}\left[g_{a}^{2}(3-\cos (\theta))\left(n_{n}+n_{p}\right) S_{A}+(1+\cos (\theta)) n_{n} S_{V}\right] .
$$

The overall effect of the corrections, due to many-body effects, is to lower the axial response function, $S_{A}$, and therefore lower the total neutral-current scattering cross section. Since the Virial EOS is only valid at low densities, we transition to a model-dependent expression for $S_{A}$ at high densities, which is based on the random phase approximation (RPA) work of Burrows and Sawyer (1998). We empirically fit our results with the 
following expression for use in our simulations.

$$
S_{A}^{f}\left(n, T, Y_{e}\right)=\frac{1}{1+A\left(1+B e^{-C}\right)},
$$

where

$A\left(n, T, Y_{e}\right)=A_{0} \frac{n\left(1-Y_{e}+Y_{e}^{2}\right)}{T^{1.22}} ; B(T)=\frac{B_{0}}{T^{0.75}} ; C\left(n, T, Y_{e}\right)=C_{0} \frac{n Y_{e}\left(1-Y_{e}\right)}{T^{0.5}}+D_{0} \frac{n^{4}}{T^{6}}$,

where $n$ is the baryon density in units of nucleons $/ \mathrm{fm}^{3}, T$ is the matter temperature in $\mathrm{MeV}$, and $Y_{e}$ is the electron fraction. We take as constants, $A_{0}=920, B_{0}=3.05$, $C_{0}=6140$, and $D_{0}=1.5 \times 10^{13}$. We note that setting $B_{0}=0$ removes the Virial EOS contributions to the neutrino response and effectively reduces our expressions for the axial response function to the values from Burrows and Sawyer (1998). As noted in Horowitz et al. (2017), at low densities, where the Virial EOS is valid, the Virial contributions decrease $S_{A}$ up to a factor of 2 more than the contributions from Burrows and Sawyer (1998). We refer the reader to Figure 2 of Horowitz et al. (2017) for a graphical display of $S_{A}$ and $S_{A}^{f}$ for different $\rho, T$, and $Y_{e}$ values and for various neutrino interaction assumptions.

The goal of these proceedings is to explore the effect of these Virial contributions, as well as the many-body effects in general, on the core-collapse supernova dynamics. To this end, we perform a large number of simulations, broken into two parts. First, we simulate core-collapse in four progenitor models from Woosley and Heger (2007): s12WH07, s15WH07, s20WH07, and s25WH07. For each progenitor we run a simulation with and without the corrections from Horowitz et al. (2017). Second, for model s20WH07 we simulate an additional 15 simulations, split into three sets: without corrections, with only high density corrections (i.e. $B_{0}=0$ ), and with full corrections. In an attempt to remove the effect of stochastic motions on our results and interpretations, for each of the three sets we do five simulations, each one starting with different random perturbations (at the $0.1 \%$ level) to the density field. We apply these perturbations everywhere at the start of the 2D simulation. Each simulation is started at $15 \mathrm{~ms}$ after bounce. We take the evolution up to $15 \mathrm{~ms}$ from a 1D GR1D simulation (O'Connor 2015).

\section{Results}

\subsection{Progenitor Dependence}

We simulate core-collapse in four progenitors, with and without the neutral-current scattering cross section corrections discussed above. We show the evolution of the shock radius in Figure 1. Dark colours represent the simulations without the corrections, while the lighter colours are the corresponding simulations with corrections. In all cases the shock radius propagates out to $\sim 150 \mathrm{~km}$, stalls, and recedes. For models s15WH07, s20WH07, and s25WH07, the reduced neutral-current scattering cross sections lead to earlier explosions. Explosions times are reduced by $\sim 100 \mathrm{~ms}-150 \mathrm{~ms}$. For model s12WH07, the corrections lead to a late-time explosion where there was not one observed in the model without corrections. The impact of these corrections is more modest than determined by Burrows et al. (2016), where the inclusion of these corrections dramatically decreased the explosion times in several models.

\section{2. s20WH07}

Two dimensional simulations of core-collapse supernovae are sensitive to stochastic variations in the matter motions during the accretion phase. This can lead to differences in 


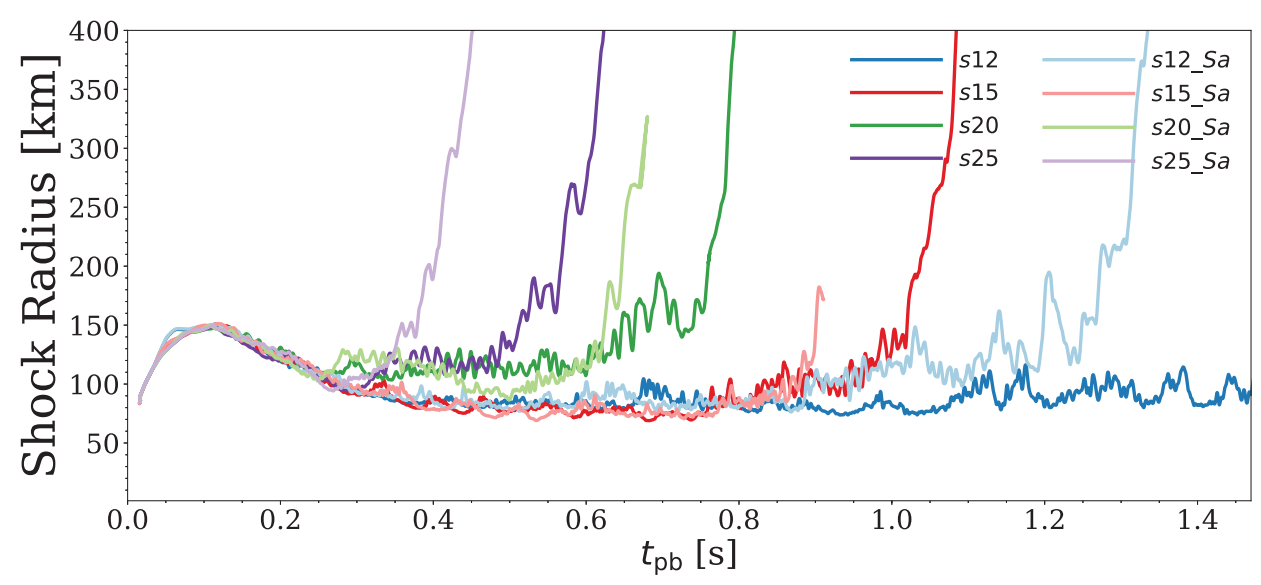

Figure 1. Shock radius evolution for four different progenitors, with and without the explored corrections from Horowitz et al. (2017). The corrections lead to earlier explosions.

explosion times which could potentially cloud interpretations. To overcome this, and to probe the effect of the corrections discussed in $\S 2$ more deeply, we perform many simulations with the s20WH07 model. As mentioned above, we run 15 simulations in total and explore three unique setups. Each simulation is started with a different set of random perturbations on the density field $(\rho \rightarrow \rho \times(1+r)$; where $r$ is a different random number for each zone between -0.001 and 0.001$)$. The first set of five simulations ignores any corrections to the neutral-current scattering cross section from the axial response, i.e. $S_{A}=1$ in Eq. 2.1. The second set of simulations uses $S_{A}$ as shown in Eq. 2.2 but sets $B_{0}=0$ and the third set used Eq. 2.2 directly. We show the evolution of the shock radius in these 15 simulations in Figure 2. We colour-code each set, but do not distinguish between the five runs of each set for clarity. We see the same results as the previous section. The reduced value of $S_{A}$ due to many-body effects leads to earlier explosions. We define the explosion time to be when the shock crosses $400 \mathrm{~km}$. With this definition, the average explosion times, and the variances are determined to be:

$$
t_{\exp }^{S_{A}=1}=(723 \pm 63) \mathrm{ms} ; \quad t_{\exp }^{S_{A}^{f}, B_{0}=0}=(597 \pm 37) \mathrm{ms} ; \quad t_{\exp }^{S_{A}^{f}}=(583 \pm 42) \mathrm{ms} .
$$

The key observation we make here is that the Virial EOS contributions to the axial response, which lower $S_{A}$ at lower densities, do not have a significant effect on the explosion dynamics. Statistically the explosion times are indistinguishable from those that only include the reduction due to the effective RPA corrections. We explore this in more detail by examining the neutrino quantities.

In Figure 3, we show graphs of various neutrino quantities over the first $600 \mathrm{~ms}$ of evolution for the three different sets of simulations. For each set we average the quantities for the five different simulations. This significantly reduces the scatter and allows us to see the effect of each neutrino opacity change in detail. In the upper left panel we show the luminosity for each of the three neutrino species and for each set of simulations. The largest effect we see is the increase in the $\nu_{x}$ luminosity, by $\sim 10 \%$, when the many-body corrections to the axial response function are considered. The lower total scattering rates that result from the lower $S_{A}$ allow the $\nu_{x}$ neutrinos to escape more easily and results in more cooling. Consequently, this leads to higher $\nu_{x}$ average energies (upper right panel) and faster contracting protoneutron star radii (bottom right panel). The faster contraction has the side effect of increasing the electron type neutrino and anti-neutrino luminosities and average energies which leads to increased neutrino heating (bottom left 


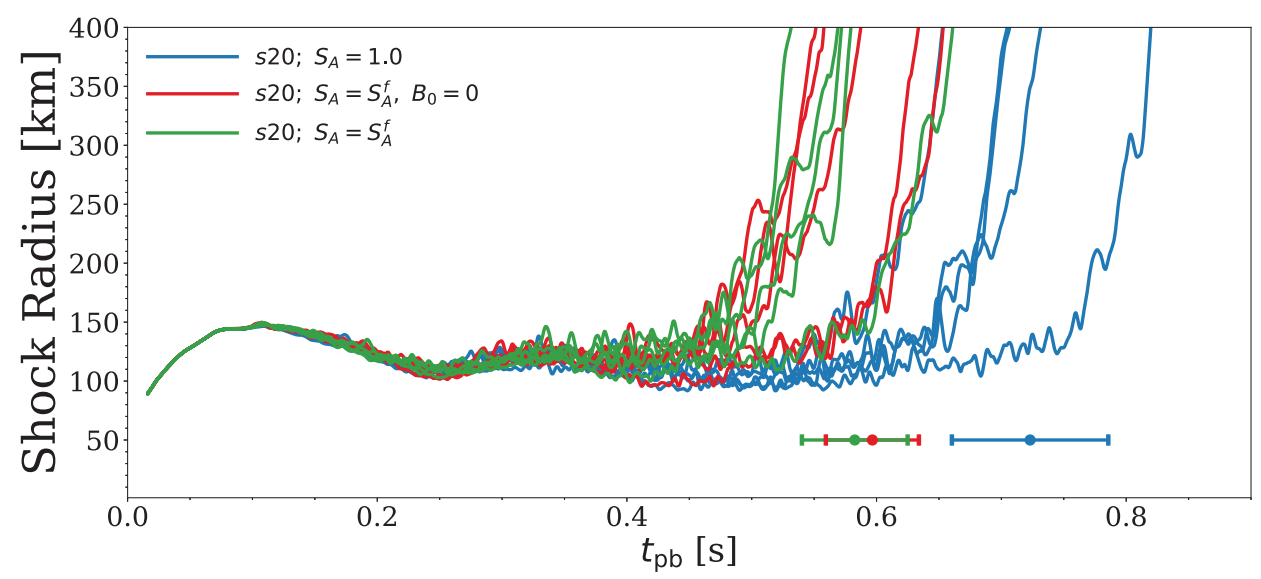

Figure 2. Shock radius evolution for 15 simulations of s20WH07 using three different neutrino-nucleon neutral current scattering cross sections based on Eq. 2.1. Five use $S_{A}=1$ (blue), five take $S_{A}=S_{A}^{f} ; B_{0}=0$ (red), the remaining five use $S_{A}=S_{A}^{f}$ (green). We include the average explosion time and the variance within the five simulations.

panel). This is the same effect observed in Melson et al. (2015) when they reduced the neutral-current scattering by considering relatively large strange-quark contributions.

Here we notice that the Virial contributions do indeed lead to more cooling, higher energies, and faster contraction compared to the $B_{0}=0$ simulation, as expected. However, the additional effect is relatively small compared to the impact the Virial contributions have on $S_{A}$ (upwards of a factor of 2 more important than the RPA corrections at low densities). We can resolve this apparent conflict by considering the properties of the region where the $\nu_{x}$ 's are emitted from and scatter through and comparing those to the region where the Virial contributions dominate. Unlike $\nu_{e}$ and $\bar{\nu}_{e}, \nu_{x}$ do not undergo charged-current interactions. The dominant emission process is via pair production. Due to the temperature dependence, the luminosity build up occurs deeper (and at higher densities, $\rho \sim 10^{12}-10^{13} \mathrm{~g} \mathrm{~cm}^{-3}$, and temperatures $T \sim 10 \mathrm{MeV}$ ) in the protoneutron star. At these densities and temperatures the corrections to $S_{A}$ in Eq. 2.2, which are of order $\sim 20 \%$, are either dominated by the effective RPA (at the highest densities) or of similar value between the Virial and effective RPA. These two reasons explain why the Virial EOS contributions to the axial response do not significantly affect the core-collapse supernova dynamics.

\section{Conclusions}

In this proceedings for IAUS 331, we present a detailed look at the effect of corrections to the neutral-current neutrino-nucleon scattering cross sections on the core-collapse supernova explosion dynamics. In particular, we look at recent model-independent corrections to the axial response $\left(S_{A}\right)$ of low-density nuclear matter described by the Virial EOS. We argue here that corrections to $S_{A}$ at the level of $\sim 20 \%$ can influence the dynamics, however, at the relevant densities $\left(10^{12}-10^{13} \mathrm{~g} \mathrm{~cm}^{-3}\right)$ and temperatures $(\sim 10 \mathrm{MeV})$ our model-independent calculation begins to break down and we must resort to modeldependent, RPA calculations to estimate $S_{A}$. Due to the important role these corrections may play in the evolution of the central engine of core-collapse supernovae, we advocate for a better understanding of the neutrino response at these densities. 

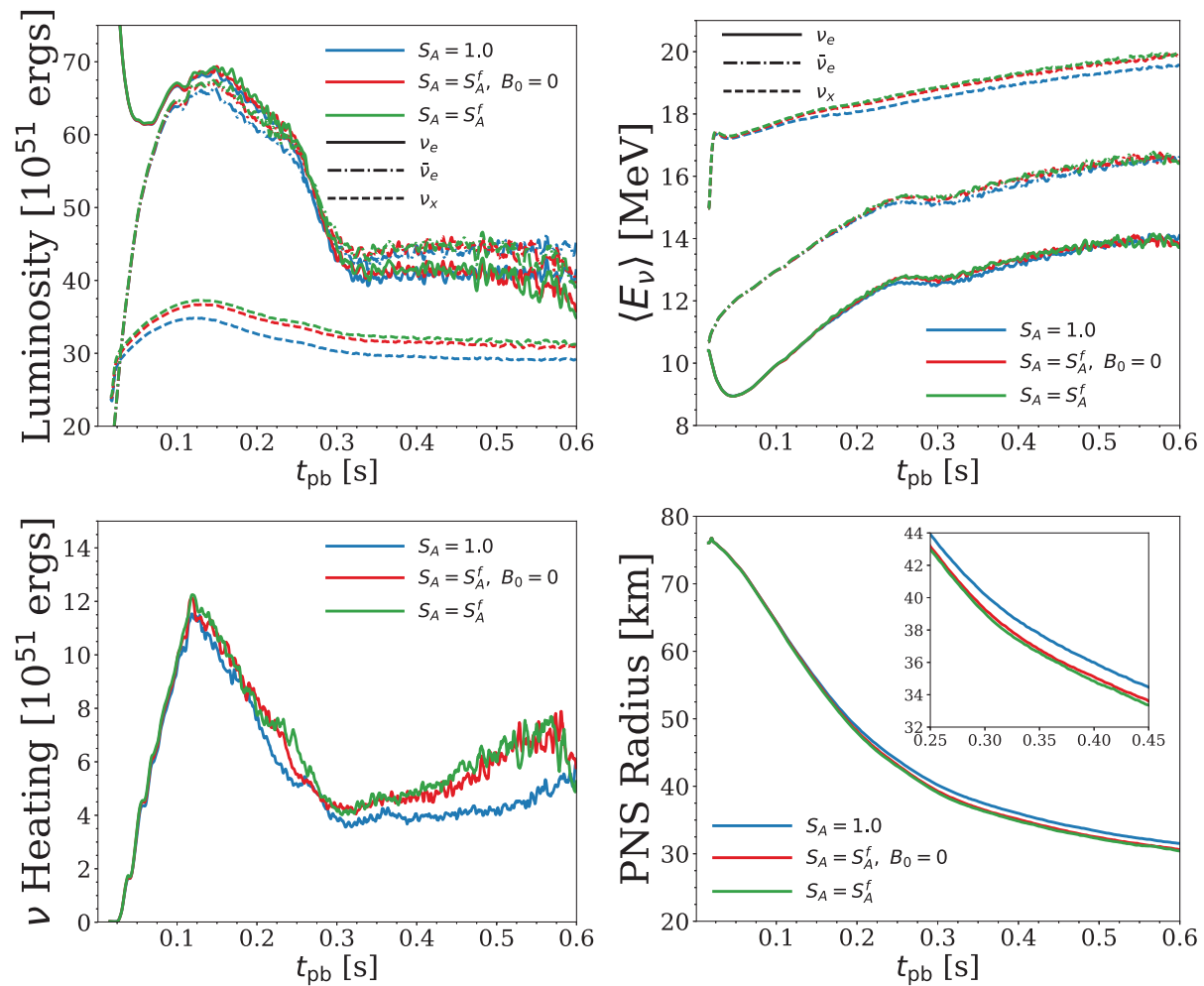

Figure 3. Neutrino related quantities for model s20WH07. Here we show the neutrino luminosity (top left) and average energy (top right) for each neutrino species. We also show the net neutrino heating (bottom left) and the protoneutron star radius (bottom right; defined as the radius of $\rho=10^{11} \mathrm{~g} \mathrm{~cm}^{-3}$ ). For each of the three configurations studied, we average the quantities from the five simulations in order to reduce scatter and better ascertain the overall effect.

Acknowledgements: We thank O.L. Caballero and A. Schwenk for collaborative work on the Virial EOS. Support was provided by NASA through Hubble Fellowship grant \#51344.001-A awarded by the STScI, which is operated by the AURA, for NASA, under contract NAS 5-26555. Computations were performed on the Zwicky cluster at Caltech, supported by the Sherman Fairchild Foundation and by NSF award PHY-0960291, and on XSEDE which is supported by National Science Foundation grant number ACI-1548562.

\section{References}

Bruenn, S. W. (1985). ApJS, 58:771-841.

Burrows, A. \& Sawyer, R. F. (1998). PRC, 58:554.

Burrows, A., Vartanyan, D., Dolence et al. (2016). ArXiv e-prints: 1611.05859.

Couch, S. M. \& O'Connor, E. P. (2014). ApJ, 785:123.

Fryxell, B., Olson, K., Ricker, P., et al. (2000). ApJS, 131:273.

Horowitz, C. J. (2002). PRD, 65(4):043001.

Horowitz, C.J., Caballero, O.L., Lin, Z., et al. (2017). PRC, 95(2):025801.

Melson, T., Janka, H.-T., Bollig, R., Hanke, F., Marek, A., \& Müller, B. (2015). ApJL, 808:L42.

O'Connor, E. (2015). ApJS, 219:24.

O'Connor, E. \& Couch, S. M. (2015). submitted to ApJ; arXiv:1511.07443.

Lattimer, J. M. \& Swesty, F. D. (1991). Nuc. Phys., A535:331.

Woosley, S. E. \& Heger, A. (2007). Phys. Rep., 442:269. 